\title{
Multi-Robot Coordination and Planning in Uncertain and Adversarial Environments
}

\author{
Lifeng Zhou • Pratap Tokekar
}

Received: date / Accepted: date

\begin{abstract}
Purpose of review Deploying a team of robots that can carefully coordinate their actions can make the entire system robust to individual failures. In this report, we review recent algorithmic development in making multi-robot systems robust to environmental uncertainties, failures, and adversarial attacks.
\end{abstract}

Recent findings We find the following three trends in the recent research in the area of multi-robot coordination: (1) resilient coordination to either withstand failures and/or attack or recover from failures/attacks; (2) risk-aware coordination to manage the trade-off risk and reward, where the risk stems due to environmental uncertainty; (3) Graph Neural Networks based coordination to learn decentralized multi-robot coordination policies. These algorithms have been applied to tasks such as formation control, task assignment and scheduling, search and planning, and informative data collection.

Summary In order for multi-robot systems to become practical, we need coordination algorithms that can scale to large teams of robots dealing with dynamically changing, failure-prone, contested, and uncertain environments. There has been significant recent research on multirobot coordination that has contributed resilient and risk-aware algorithms to deal with these issues and reduce the gap between theory and practice. Learningbased approaches have been seen to be promising, es-

Lifeng Zhou

Electrical \& Computer Engineering, Virginia Tech

Blacksburg, VA 24061, USA

E-mail: lfzhou@vt.edu; lfzhou@seas.upenn.edu

Pratap Tokekar

Computer Science, University of Maryland

College Park, MD 20742, USA

E-mail: tokekar@umd.edu pecially since they can learn who, when, and how to communicate for effective coordination. However, these algorithms have also been shown to be vulnerable to adversarial attacks, and as such developing learning-based coordination strategies that are resilient to such attacks and robust to uncertainties is an important open area of research.

Keywords Multi-robot systems · Resilient and risk-aware coordination · Graph neural networks

\section{Introduction}

As the famous adage goes "there is safety in numbers." The objective of this paper is to give an overview of the recent research that is aimed at making teams of robots safer and robust through careful coordination. The last decade has seen multi-robot systems being increasingly used in areas such as manufacturing, warehouse management, agriculture, and environmental monitoring [1, 2]. Deploying a team of robots, instead of a single one, increases the spatial and temporal scales at which the robots are deployed. However, beyond improving the scalability, a team of robots also improve the robustness of the entire system through redundancy and heterogeneity. Through careful coordination between the actions of the robots, we may be able to make the team as a whole robust to uncertainty present in the real-world and to individual failures [3, 4, This is a crucial capability as we are starting to have robots coexist with humans and therefore, we need algorithms that can be trusted under real-world, not just ideal conditions. In this paper, we report recent progress in multi-robot coordination and planning that is particularly focusing on increasing the resilience and robustness of the system. 
Designing such algorithms, especially to enable coordination between multiple robots under uncertainty and robot failures is a challenging problem. Due to noisy sensing [5, imperfect motion, unknown environmental conditions [6, 7, or robot failures [8, the robots are very likely to encounter unforeseen events in practice. For example, the travel time for the robots may depend on environmental factors such as traffic and may not be exactly known. As a result, any algorithm that coordinates the actions of the robots will have to operate on uncertain information. Coordination in the presence of uncertainty can be risky [6, 7, 9]. There is a risk of the actual performance of the robot team during execution time being significantly different from the expected performance during planning time.

In addition to uncertainty, it is also challenging to deal with catastrophic robot failures either due to natural causes or due to adversarial attacks [10, 11, 12. For example, an adversary can attack the system by spoofing fake identities [13] or sharing incorrect information [14]. An adversary may also choose specific robots to attack so that the team encounters a worst-case loss in the performance [15. This is especially crucial in applications such as surveillance and security where counter-Unmanned Aerial Vehicles strategies are being developed 16. In many cases, even if a part of the system fails, the entire system performance can be significantly undermined [3, 4].

Pioneering work in this area was done by Parker 17 . who proposed the ALLIANCE framework for coordination in heterogeneous robot teams where individual robots may fail. In this report, we discuss the recent trends, building on this pioneering work, in designing effective multi-robot coordination approaches to address the challenges mentioned above. Specifically, we focus on three recent trends:

1. Resilient coordination algorithms have been designed to counter adversarial attacks and failures (Section 22). These algorithms can be roughly categorized into two broad approaches. One, researchers focused on designing robust coordination approaches that prepare systems to withstand attacks 4, 18, (Section 2.1 and 2.2). Two, adaptive and reactive algorithms have been proposed to enable systems to recover from attacks and failures [19, 20] (Section 2.3. Resilient coordination algorithms have shown the effectiveness in many robotics tasks, such as team formation [18, target estimation [21, and data collection [4, 15].

2. Risk-aware coordination aims at addressing the risk of performance loss from uncertainty when a team of robots are operating in uncertain environments (Section 3). One line of recent work is focused on defining and using appropriate risk measures of the stochastic team objective (induced by the uncertainty). Several risk-aware coordination algorithms have been developed that allow robot teams to tradeoff the reward collected with the risk due to uncertainty [8, 22, 23] (Section 3.1 and 3.2). Another line of work is focused on designing risk/uncertaintyaware search algorithms for multi-robot planning with uncertainty 24] (Section 3.3). These risk-aware coordination algorithms have been shown to be useful in many robotics tasks, such as team formation [8, 25], task allocation [22], data collection [23], and graph search in partially known environments 24 .

3. Coordination using Graph neural networks (GNNs) is a relatively new research direction in multi-robot coordination. By leveraging useful inherent properties of GNNs such as decentralized information propagation, permutation equivalence, and stability [26], researchers have shown how to learn decentralized and optimal strategies for multi-robot tasks such as team formation [27, path planning [28, and task scheduling and assignment 29] (Section (4). One significant advantage of GNN-based approaches is that the strategies learned from smaller cases can be generalized to larger settings. For example, these strategies show promise in generalizing across the number of robots 30, 31] which can help make the team robust to individual failures. These advantages enable GNN based methods to provide near-optimal solutions for large-scale coordination problems, which might be otherwise intractable by using classical coordination approaches.

Based on these research trends, we also discuss several potential research directions for the near term. These include security in deep-learning based coordination methods, risk-aware coordination with the trade-off between local and global interest, and parsimonious communications in GNN based coordination approaches (described further in Section 5 .

\section{Resilient Coordination}

Resilience to unexpected events is a critical capability for getting robots from controlled factory settings to the real world [32. Resilience broadly refers to two capabilities: (1) preparing a system to be robust enough to withstand faults and attacks; and (2) adapting and recovering from individual failures and attacks. There has been significant work focusing on making individual robots resilient [33, 34. Recently, there is a trend on investigating resilience in multi-robot teams, often grounded in tasks such as formation control 14, 18, 35. 
36, 37, 38, 39, 40, 41, 42, 43, 44, wireless communication [13, 45], state estimation [21, 46, 47, 48, data collection [4, 15, 49, 50, 51, attack-defense games 52, [53, 54, and adaptive reconfiguration [19, 20, 55, 56]. A summary of these applications is found in Table 1

A general goal in resilient multi-robot systems is to guarantee a desired team performance even though some robots in the team fail [20, are malicious 14, or get attacked [4]. We summarize the sources of robot failures and attacks in Table 2. To address these challenges, researchers have been focusing on designing various coordination approaches to make multi-robot teams resilient. We describe the main approaches in the following.

\subsection{Resilient Formation Control and Estimation}

Formation control is a classical problem for multi-robot systems. The goal is for the robots to achieve a desired formation, maintain it as the formation moves, and reconfigures to a new formation [59]. The standard assumption in classical techniques is that all robots are cooperative. Saulnier et al. presented a resilient formation control approach [14 where they designed a control policy that enables a team of mobile robots to achieve desired formations even though some team members are non-cooperative (or adversarial) in the sense that they broadcast malicious/spoofing signals. The authors built on the previous work about the $r$ robustness of communication topology [35] and proposed an efficient approach to manage the algebraic connectivity of the graph to guarantee resilient formation. Following this line, resilient formations have been investigated in time-varying communication graphs [18, triangular robust graphs [37, and triangular and square lattices [38, 39. Then Usevitch and Panagou studied a resilient leader-follower formation problem in [41] where they proposed a resilient controller that allows wellbehaving robots to track a reference state even though a bounded subset of leaders and followers are adversarial. In these studies, the robots update their motion synchronously and periodically. As a contrast, some recent work has focused on the problem of resilient formation if the robots have their own clocks and may update in an asynchronous way, which makes progress towards designing resilient event-triggered and self-triggered coordination strategies [43, 44].

In addition to resilient formations, researchers have investigated resilient state estimation of a static or dynamical process (target of interest) by a team of robots [21, 46, 47, 48. In particular, Mitra et al. presented an important approach 21] where robots are tasked to estimate the state of a dynamical process given the challenges of intermittent measurements, communication loss, and adversarial team members. To cope with these challenges, they designed resilient, distributed algorithms to guarantee correct state estimation in the dynamically changing and adversarial environments.

\subsection{Resilient Submodular Maximization}

State estimation, described in the previous subsection, belongs to a broader class of information gathering problems. These problems include process or target tracking 60, 61, 62, 63, 64, environmental monitoring 65, and search-and-rescue 66. In multi-robot information gathering problems, the objective is to maximize the joint information gathered by the robot team, such as the joint area covered or explored. Given that the robots may have overlaps over the collected data, this objective generally turns out to be a submodular function 67, 68.

Submodularity captures the property of diminishing returns of set fucntions. Even though maximizing submodular functions is NP-hard, a simple greedy algorithm yields a constant-factor approximation of the optimal solution [67, 68. However, if the robots operate in an adversarial environment and some of them or their sensors are attacked, the simple greedy algorithm may perform arbitrarily bad [4. To this end, Tzoumas et al. presented the first scalable resilient algorithm to counter any number of adversarial (specifically, denial-of-service) attacks or failures [69. Building on this work, recent studies have investigated designing resilient information collection algorithms in target tracking 4 and information gathering scenarios [15. Particularly, the resilient target tracking algorithm proposed by Zhou et al. 44 guarantees a provably close-to-optimal team performance even though some robots in the team are attacked and their tracking cameras are blocked (Figure 1). Then the same set of authors extended their resilient target tracking algorithm to a constrained communication scenario where robots have limited communication range and can only communicate locally [50. They proposed a distributed robust algorithm where robots first form local groups and then the groups operate the resilient target tracking algorithm [4] in parallel to counter attacks. Later, Shi et al. applied the resilient algorithm [69] into a resilient team orienteering problem where a group of robots plans paths within a limited budget to collect data in an adversarial environment [51]. The authors designed a robust multi-path planning algorithm that allows robots to plan trajectories over a longer time horizon and guarantees provable team data-collection 
Table 1: Resilient multi-robot coordination: tasks

\begin{tabular}{|c|c|}
\hline Formation Control & {$[1,18,36,37,38,39,40,41,42,43,44$} \\
\hline Task Allocation & 57 \\
\hline Target Tracking & $46,47,21,48,4,15,50,55,58$ \\
\hline Coverage/exploration & $15,56,51$ \\
\hline Communication & 55, 58, $13,45,19$ \\
\hline Perimeter defence & $52,53,54$ \\
\hline
\end{tabular}

Table 2: Resilient multi-robot coordination: malfunction sources

\begin{tabular}{|c|c|}
\hline Spoofing Attack & {$[13,14,18,21,36,37,38,39,40,41,42,43,44,46,47,48$} \\
\hline Robot/Resource Failure & $19,45,55,58$ \\
\hline Denial of Service Attack & $4,15,50,51$ \\
\hline Environmental Change & 57 \\
\hline
\end{tabular}

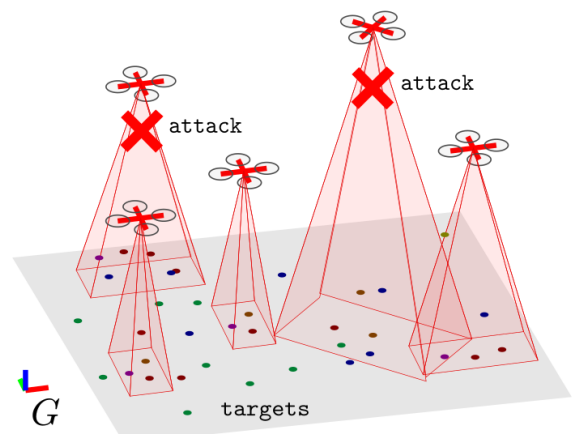

Fig. 1: Multi-robot target tracking with adversarial attacks. An adversary can block robots' tracking cameras to disable their tracking abilities. (C) [2020] IEEE. Reprinted, with permission, from [50]).

performance, despite a number of robot being attacked or failing.

\subsection{Resilient Reconfiguration via Optimization}

In addition to designing coordination algorithms that withstand attacks or failures [4, 50, 51, 69, we also need resilient reconfiguration approach that enables robot teams to adaptively recover after attacks or faults [19, 20, 55, 56. Ramachandran et al. studied the problem of maintaining resource availability in a network of multiple robots [19] in such conditions. The resources can be sensing or computational capabilities provided by the robots. The authors designed a resilient resource reconfiguration framework that enables robots to maintain access to the resources by effectively reconfiguring inter-robot communication networks if their resources are not available due to failures. Then this resilient reconfiguration framework was utilized for maintaining sensing quality for robots to track targets [55]. Later, a resilient multi-robot coverage framework was designed in 56] where well-functioning robots adaptively reposition themselves to maintain a good team coverage performance once a robot in the team fails. Further, to completely cover or explore an environment by a team of robots, Song et al. presented a distributed eventdriven replanning algorithm to adaptively assign tasks to compensate for the team loss induced by robot failures [70. Specifically, a game-theoretic structure was designed to trigger resilient task reallocations for the well-behaving robots, e.g., either keeping performing their own tasks or helping the failed robot to perform its task.

\section{Risk-Aware Coordination}

The resilient coordination approaches discussed heretofore seek to optimize for the worst-case performance. While in most cases, the focus is on being resilient to adversarial attacks, these approaches can also be used to find a conservative plan that is resilient to uncertainty that is present in most practical settings. However, optimizing for the worst-case may be too conservative in such settings. Instead, one may want approaches that can trade-off reward versus risk due to uncertainty. The uncertainty can stem from imperfect information about internal information about the robots, noisy sensing, imperfect motion [5] as well as from external sources such as unknown or partially known environments 23 7. A summary of various sources of uncertainty is given in Table 3 . Because of the uncertain information, the actual robot system's performance in execution can significantly diverge from the expected performance at the planning stage, which puts the system's performance at risk.

The standard approach of dealing with uncertainty is to consider either the expected-case performance 6 


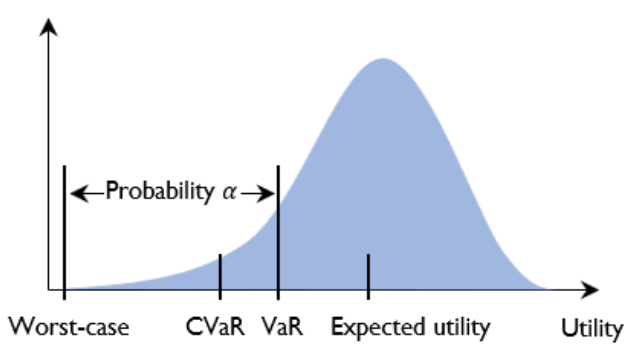

Fig. 2: An illustration of several risk measures.

71. or the worst-case performance 62, 63, 72. These represent extremes; recently, there is a trend of using more nuanced measures that better capture risk. In particular, these include mean-variance [7, 73], chanceconstraints or Value-at-Risk (VaR) 22, and ConditionalValue-at-Risk (CVaR) [74, 75, 23] (Figure 2). Specifically, the mean-variance measure has the form $\mathbb{E}(U)+$ $\lambda \sigma^{2}(U)$ where $\mathbb{E}(U)$ and $\sigma^{2}(U)$ are the expectation and the variance of the stochastic performance (or utility) $U$, and $\lambda$ is a weighting parameter deciding the relative importance we place on the expected performance and the risk (in this case, the variance). The Value-at-Risk $\operatorname{VaR}_{\alpha}(U)$ denotes the $\alpha$-quantile of stochastic utility $U$ and is defined as:

$$
\operatorname{VaR}_{\alpha}(U)=\inf \{\tau \in \mathbb{R}, \operatorname{Pr}[U \leq \tau] \geq \alpha\},
$$

with $\alpha \in[0,1]$ denoting the user-prescribed risk tolerance level. Based on VaR, the Conditional-Value-atRisk $\operatorname{CVaR}_{\alpha}(U)$ is defined as:

$$
\mathrm{CVaR}_{\alpha}(U)=\underset{y}{\mathbb{E}}\left[U \mid U \leq \operatorname{VaR}_{\alpha}(U)\right]
$$

where $y$ is a random parameter representing the uncertainty. Thus, CVaR measures the expected performance in the $\alpha$ worst fraction of cases. A summary of commonly used risk measures with a comparison in terms of six proposed axioms has been described in 76 .

Based on these risk measures, researchers have developed risk-aware approaches in various robotics tasks such as graph search and motion planning [74, 75, 7, 77, controls [8, 78, 25], task allocation and assignment [6, 22, 79, 80, information collection [81, 23], and machine learning [75, 82. Here, we focus on the risk-aware approaches for multi-robot systems. We describe these approaches in the following and summarize the corresponding applications in Table 4.

\subsection{Risk-Aware Control and Optimization}

Researchers have recently investigated risk-awareness in multi-robot control and optimization such as formation control [8], chance-constrained optimization [25, 22], and CVaR based optimization [83]. Particularly, Park and Hutchinson designed distributed robust controllers to guarantee the rendezvous for multi-robot systems even though some robots in the team randomly fail 8. They considered both the mean-variance and the worst-case measures for the stochastic cost function. Zhu and Alonso-Mora used a chance-constraint measure, explicitly constraining the probability of an undesirable event, to guarantee the safe navigation (in a probabilistic sense) of micro-air vehicles in cluttered and dynamically changing environments [25]. They formulated a chance-constrained nonlinear model predictive control problem that takes the probability of collision as constraints. For this problem, they proposed three coordination strategies based on different communication settings and evaluated the effectiveness of proposed methods through real-world experiments. Later, the authors utilized buffered uncertainty aware Voronoi cells, computed by satisfying a set of chance constraints, to guarantee the inter-robot collision avoidance in a probabilistic way [84. The chance constraints have also been utilized in non-convex collision-free path planning 85. and for stochastic planning problems that go beyond the classical Gaussian uncertainty [86].

Risk-awareness has also been considered in task assignment problems. Yang and Chakraborty studied a chance-constrained combinatorial optimization problem that takes into account the risk in multi-robot assignment [22]. They later extended the chance-constrained formulation to knapsack problems 79. They solved the problem by transforming it into a risk-aware problem with mean-variance measure [8]. Instead of chance-constrained measure, Nam and Shell analyzed the sensitivity of assignment optima by using both the expectation and CVaR measures in a multi-robot task allocation problem 83 .

\subsection{Risk-Aware Submodular Maximization}

As described in Section 2.2, many multi-robot coordination objectives are naturally submodular. Existing work on submodular optimization has focused on the deterministic case; however, in practice the objective can be stochastic (e.g., random robot or sensor failures 8, 23. or uncertain travel time because of unknown traffic $[23,6]$ ).

Using expectation as the measure, Prorok studied the problem of assigning multiple robots to goal locations under travel-time uncertainty [6]. This approach assigned redundant robots to goal locations to counter uncertainty, which makes the objective, the total waiting time at goal locations, a supermodular function 
Table 3: Risk-aware multi-robot coordination: uncertainty sources

\begin{tabular}{|c|c|}
\hline $\begin{array}{l}\text { Random Robot/Sensor Failure } \\
\text { Uncertain Travel Time/Distance } \\
\text { Dynamic or Unknown Obstacles } \\
\text { Generic Partial Observability } \\
\text { Extraction Uncertainty by DL }\end{array}$ & 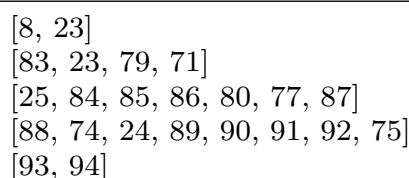 \\
\hline
\end{tabular}

Table 4: Risk-aware multi-robot coordination: tasks and techniques

\begin{tabular}{|c|c|}
\hline Formation control & 8 \\
\hline Task assignment & $83,22,79,80,94$ \\
\hline Coverage/exploration & $23,81,7$ \\
\hline Collision avoidance & $25,84,85,86$ \\
\hline Graph search & $80,81,7,77,88,74,24,89,90,91,87,71,93,94$ \\
\hline Learning & $93,94,92,75,82$ \\
\hline
\end{tabular}

$(f(\mathcal{S})$ is supermodular if $-f(\mathcal{S})$ is submodular). Since the expectation of a supermodular function is still supermodular, a simple greedy algorithm was utilized to efficiently minimize the total waiting time. The expectation was also used to measure the stochastic submodular team performance in a team surviving orienteering problem where a team of robots is tasked to traverse a dangerous environment with a probability of survival for each robot [81]. The authors proposed an approximate greedy approach with a provable guarantee for selecting risk-aware paths for the robots. They also investigated the problem in an online version and with heterogeneous teams and verified the proposed algorithm in large-scale real-world scenarios.

While optimizing the expected performance has its uses, it also has its pitfalls since the expectation measure is risk-neutral and may not work well in some extreme (bad) cases [23, 76]. At the same time, decisions made considering worst-case scenarios can be conservative 62, 63, 4]. Recent work optimize for risk measures such as CVaR and aim to fill this gap between expected-case and worst-case analysis 95, 76. By optimizing CVaR with a user-defined risk parameter $\alpha$, the robot team can balance the trade-off between the reward and the risk it would like to take 23 .

However, when the objective is a discrete (stochastic) submodular function, Maehara presented a negative result for optimizing CVaR [96] - there is no polynomial-time multiplicative approximation algorithm under some reasonable assumptions in computational complexity. To circumvent this issue, Ohsaka and Yoshida adopted an idea from portfolio optimization and proposed a method of selecting a distribution over available sets rather than selecting a single set, and gave a provable guarantee [97. Following this line, Wilder considered a CVaR maximization of a continuous submodular function instead of the submodular set functions
98. They gave a $(1-1 / e)$-approximation algorithm for continuous submodular functions and also evaluated the algorithm for discrete submodular functions using portfolio optimization [97. Instead of selecting a portfolio over the available sets, Zhou and Tokekar presented a risk-aware sampling-based algorithm with a bounded approximation to select a single set for CVaR based discrete submodular optimization [23, 99. They demonstrated the effectiveness of the risk-aware algorithm through two case studies: sensor placement with random failures and vehicle assignment for mobility-ondemand under travel-time uncertainty (with an online version presented in [99]). Their risk-aware algorithm has also been utilized for planning risk-aware paths for the robots with uncertainty extracted from Bayesian deep learning models 93,94 and extend to the case of planning a route 100 .

\subsection{Uncertainty-Aware Search and Planning}

Classical planning problems have been extensively studied 101. Based on the assumption that the robot actions and search space are deterministic, researchers have developed many powerful planning algorithms such as $\mathrm{A}^{*}$ [102 and RRT* 103 to find optimal start-to-goal paths. However, after incorporating uncertainty from the robot state and/or the world model, these planning problems become challenging [88, 7]. Some recent work has investigated incorporating the uncertainty in the classical planners to tackle these problems [104, 73].

A typical way to address the uncertainty in planning is to formulate these problems as Partially Observable Markov Decision Processes (POMDPs) that consider uncertainty in robot states, actions, and observations 105, 24. Particularly, for multi-robot coordination, Amato et al. presented a MacDec-POMDP plan- 
ning algorithm to deal with uncertain sensing and limited communication in multi-robot planning [89. They showed several properties of the proposed MacDec-POMDP planning algorithm and its advantages in solving larger problems over existing Decentralized POMDPs (DecPOMDP) planners. Later, to solve multi-robot planning problems in continuous spaces, Omidshafiei et al. extended the Dec-POMDP to the Decentralized Partially Observable Semi-Markov Decision Process (DecPOSMDP) using task macro-actions that allow robots to make decisions asynchronously [90. They also proposed scalable algorithms to generate robust solutions for solving Dec-POSMDPs. If the underlying Dec-POMDP model is not assumed to be known a priori or a full simulator is available at planning time, Liu presented a policy-based reinforcement learning approach that updates policies though agents interacting with the environment [106]. They also showed the proposed approach can generate valid macro-action controllers (used in 89 , 90, 91]) and learn optimal policies.

Other related work on multi-robot planning under uncertainty includes belief space planning for navigation in unknown environments [87, path planning with travel-time uncertainty [71, task allocation with completion uncertainty 107, and multi-task reinforcement learning under partial observability 92 .

\section{Recent Trend: Coordination by Graph Neural Networks}

There is a growing trend of using learning-based methods, in particular Graph Neural Networks (GNNs) for multi-robot coordination 31, 108. These approaches have been shown to learn what, when, and who to communicate with depending on the task at hand, instead of following a rigid communication topology [109, 110. GNNs capture the interactions among robots by modeling their coordination as a graph where robots (nodes) share information with their neighbors through communion links (edges). GNN architectures also exhibit the transferability property because of their permutation equivalence and stability, which allows the learned policies to be generalized to previously unseen scenarios [26, 111]. Researchers have recently implemented GNNs to learn decentralized and close-to-optimal solutions for the classical problems in multi-robot systems such as formation control [31, 27, 112, 113, multi-robot path planning [28, and task assignment and scheduling [29].

Particularly, some studies have investigated learning decentralized controllers for large networks of mobile robots to achieve desired formations by either using
GNNs to imitate centralized controllers with global information 27] or employ GNNs to parameterize policies Pthat are updated by policy gradients [114. Similarly, applying GNNs that allow robots to communicate with multi-hop neighbors, Li et al. have investigated generating collision-free paths for multiple robots from start positions to goal positions in cluttered environments [28. They have shown that, by imitating an expert algorithm, their learned planner that uses only local communication and observations, achieves close-to-optimal performance and is able to generalize to larger robot teams.

GNN based approach has also been proposed for solving multi-robot task scheduling and assignment that is modeled as a combinatorial optimization problem 29. Since combinatorial optimization problems are generally NP-hard, obtaining the optimal solution is computationally intractable for large-scale cases. Even though there exist many approximation algorithms (or heuristic) that runs in polynomial time, they only provide approximation guarantees or solutions without any optimality guarantee. To this end, Wang et al. combined imitation learning (i.e., imitate optimal solvers for small problems) with graph attention networks to learn fast, near-optimal scheduling that is scalable and generalizable [29].

Learning-based coordination can also lead to interesting emergent behavior in the team of robots 115 , 116, 30]. Chen et al. 30] showed emergent multi-robot behavior when learning to persistently monitor environments. Specifically, they observed that the learned policies lead to natural partitioning of the environment amongst the robots as well as lead to periodic trajectories that are to be expected in persistent monitoring. Blumenkamp and Prorok found that when a robot is trained to optimize for its own interest, it learns to communicate adversarial information to the other robots (that are optimizing the cooperative interest) [116] in coverage tasks. This opens up several interesting questions such as whether the agents can learn to be resilient to such adversarial information sharing.

\section{Conclusions}

We outlined several recent work on multi-robot coordination specifically aimed at dealing with adversaries, failures, and environmental uncertainty. While there has been significant developments in making teams of robots resilient and risk-aware, there remain several outstanding issues. In the following, we highlight three directions that we believe are going to be important going forward. 
Secure Intelligent Multi-Robot Systems The stateof-the-art deep learning based methods have shown a great promise in the field of robotics. However, when operating in safety-critical situations, these intelligent learning systems can be easily spoofed or misled (e.g., by uncertainties or adversarial attacks) which can cause unsafe situations [117, 118. Although some research has focused on designing robust deep neural networks to deal with adversarial perturbations in either the training stage or testing stage [119, 120, 121], there has been little work in the context of multi-robot coordination. As highlighted earlier, recent studies have shown emergence of adversarial communication in multi-robot reinforcement learning [116. As such, it is critical to study how to secure such multi-robot systems to adversarial attacks and guarantee a provably good team performance. Unlike say computer vision tasks, here one can leverage inter-robot coordination to mitigate such attacks as has been shown in the recent work. We need to build on this and extend it to the cases where the coordination between agents is learned instead of designed by classical algorithms.

Collaborative Decision Making with Risk Tradeoff The second future direction is to investigate how individual robots trade-off their local risks with the global/team risk to optimize team performance. For example, in a multi-robot search and rescue scenario, the robot team may want to explore the environment as much as possible while maintaining global connectivity. However, an individual robot may cares about its own safety and energy, e.g., reducing the risk of collisions or traveling through rugged terrains. To do so, it may increase the risk of disrupting team connectivity when the robot selects a safer yet far-away path (from other team members) to bypass obstacles. This can also lead to the risk of undermining team performance by affecting the decisions of other team members. That is because, to maintain global connectivity, other team members will have to abandon their high-reward paths (e.g., the paths can jointly cover a larger area), in order to move closer to this robot. Thus, there is a need for risk-aware algorithms to maximize team performance with the consideration of local and global risk tradeoff.

\section{Parsimonious Communications for Multi-Robot} Coordination using GNNs As described in Section 4. GNN based methods have shown promise in learning close-to-optimal solutions by imitating an optimal solver or an expert for multi-robot coordination [27, 28, 29]. Even though GNN based architecture only requires robots communicating with certain hop neighbors, some of these communications may not be necessary. Some studies have presented parsimonious com- munication strategies that are selective in when to communicate with neighbors [62, 63, 109] and which neighbors to communicate with 110 to cutoff unnecessary communications among neighbors. Built on this idea, the third future avenue can be embedding parsimonious communication protocols into GNN architectures for multi-robot coordination. This will be particularly important when there is heterogeneity in the sensing, actuation, and computing capabilities of the robots as well as when there are multiple tasks that the robots need to solve for. Not all tasks may require all resources. Learning task-oriented coordination is an important direction of research.

Acknowledgements The authors would like to thank the National Science Foundation (NSF IIS-1637915) and the Office of Naval Research (ONR N00014-18-1-2829) for their supports.

\section{Compliance with Ethical Standards}

Conflict of Interest The authors declare that they have no conflict of interest.

\section{Human and Animal Rights and Informed Con-} sent This article does not contain any studies with human or animal subjects performed by any of the authors.

\section{References}

Papers of particular interest, published recently, have been highlighted as:

- Of importance

• Of major importance

1. H. Christensen, "A roadmap for us robotics: From internet to robotics (2020 edition)," http:// www.hichristensen.com/pdf/roadmap-2020.pdf, September 2020, (Accessed on 11/18/2020).

2. Mobile Industrial Robots A/S, "Five collaborative mobile robots applications," https://www.mobile-industrial-robots. com/en/resources/whitepapers/ 5-collaborative-mobile-robots-applications/, Accessed: 2020-09-20.

3. V. Tzoumas, A. Jadbabaie, and G. J. Pappas, "Resilient non-submodular maximization over matroid constraints," arXiv preprint arXiv:1804.01013, 2018.

4. L. Zhou, V. Tzoumas, G. J. Pappas, and P. Tokekar, "Resilient active target tracking with multiple robots," IEEE Robotics and Automation Letters, vol. 4, no. 1, pp. 129-136, 2018. 
5. A. Martinelli, F. Pont, and R. Siegwart, "Multirobot localization using relative observations," in 2005 IEEE International Conference on Robotics and Automation (ICRA). IEEE, 2005, pp. 27972802.

6. A. Prorok, "Redundant robot assignment on graphs with uncertain edge costs," in Distributed Autonomous Robotic Systems. Springer, 2019, pp. $313-327$.

7. J. J. Chung, A. J. Smith, R. Skeele, and G. A. Hollinger, "Risk-aware graph search with dynamic edge cost discovery," The International Journal of Robotics Research, vol. 38, no. 2-3, pp. 182-195, 2019.

8. - H. Park and S. Hutchinson, "Robust rendezvous for multi-robot system with random node failures: an optimization approach," Autonomous Robots, vol. 42 , no. 8 , pp. 1807-1818, 2018, This study presents distributed robust algorithms for multi-robot rendezvous with random node failures.

9. M. J. Matarić, G. S. Sukhatme, and E. H. Østergaard, "Multi-robot task allocation in uncertain environments," Autonomous Robots, vol. 14, no. 2-3, pp. 255-263, 2003.

10. T. Denning, C. Matuszek, K. Koscher, J. R. Smith, and T. Kohno, "A spotlight on security and privacy risks with future household robots: attacks and lessons," in Proceedings of the 11th International Conference on Ubiquitous Computing. ACM, 2009, pp. 105-114.

11. N. Agmon, G. A. Kaminka, and S. Kraus, "Multi-robot adversarial patrolling: facing a fullknowledge opponent," Journal of Artificial Intelligence Research, vol. 42, pp. 887-916, 2011.

12. E. Sless, N. Agmon, and S. Kraus, "Multirobot adversarial patrolling: facing coordinated attacks," in Proceedings of the 2014 International Conference on Autonomous Agents and MultiAgent Systems. International Foundation for $\mathrm{Au}-$ tonomous Agents and Multiagent Systems, 2014, pp. 1093-1100.

13. S. Gil, S. Kumar, M. Mazumder, D. Katabi, and D. Rus, "Guaranteeing spoof-resilient multi-robot networks," Autonomous Robots, vol. 41, no. 6, pp. 1383-1400, 2017.

14. • K. Saulnier, D. Saldana, A. Prorok, G. J. Pappas, and V. Kumar, "Resilient flocking for mobile robot teams," IEEE Robotics and Automation Letters, vol. 2, no. 2, pp. 1039-1046, 2017, This study firstly introduces resilience in formation control and presents a distributed resilient controller to achieve flocking behav- iors of multi-robot systems, despite some adversarial team members.

15. B. Schlotfeldt, V. Tzoumas, D. Thakur, and G. J. Pappas, "Resilient active information gathering with mobile robots," in 2018 IEEE/RSJ International Conference on Intelligent Robots and Systems (IROS). IEEE, 2018, pp. 4309-4316.

16. Liteye, "Counter uas (cuas)," https://liteye.com/ products/counter-uas/, Accessed: 2020-09-20.

17. L. E. Parker, "Heterogeneous multi-robot cooperation," Massachusetts Inst of Tech Cambridge Artificial Intelligence Lab, Tech. Rep., 1994.

18. D. Saldana, A. Prorok, S. Sundaram, M. F. Campos, and V. Kumar, "Resilient consensus for timevarying networks of dynamic agents," in 2017 American Control Conference $(A C C)$. IEEE, 2017, pp. 252-258.

19. - - R. K. Ramachandran, J. A. Preiss, and G. S. Sukhatme, "Resilience by reconfiguration: Exploiting heterogeneity in robot teams," in 2019 IEEE/RSJ International Conference on Intelligent Robots and Systems (IROS). IEEE, 2019, pp. 6518-6525, The first work to guarantee multi-robot resilience by exploiting resource heterogeneity and reconfiguring communication networks after resource failures.

20. • J. Song and S. Gupta, "Care: Cooperative autonomy for resilience and efficiency of robot teams for complete coverage of unknown environments under robot failures," Autonomous Robots, vol. 44, no. 3, pp. 647-671, 2020, This study presents a game-theoretical strategy that trade-offs resilience and efficiency in multi-robot coverage.

21. - A. Mitra, J. A. Richards, S. Bagchi, and S. Sundaram, "Resilient distributed state estimation with mobile agents: overcoming byzantine adversaries, communication losses, and intermittent measurements," Autonomous Robots, vol. 43, no. 3, pp. 743-768, 2019, This study develops resilient, fully-distributed, and provably correct algorithms for estimating the state of a target of interest in dynamic, failureprone, and adversarial environments.

22. - F. Yang and N. Chakraborty, "Algorithm for optimal chance constrained linear assignment," in 2017 IEEE International Conference on Robotics and Automation (ICRA). IEEE, 2017, pp. 801808, This study formulates the multi-robot task assignment with payoff uncertainty as a chance-constrained combinatorial optimization problem and presents provably- 
good algorithms for solving this problem.

23. - L. Zhou and P. Tokekar, "An approximation algorithm for risk-averse submodular optimization," in International Workshop on the Algorithmic Foundations of Robotics. Springer, 2018, pp. 144-159, This study presents the first polynomial-time algorithm with bounded guarantees for solving CVaR based discrete submodular maximization problems and verifies the performance of the proposed algorithm in the multi-robot assignment and environmental monitoring scenarios.

24. F. A. Oliehoek, C. Amato et al., A concise introduction to decentralized POMDPs. Springer, 2016, vol. 1.

25. H. Zhu and J. Alonso-Mora, "Chance-constrained collision avoidance for mavs in dynamic environments," IEEE Robotics and Automation Letters, vol. 4, no. 2, pp. 776-783, 2019.

26. •• L. Ruiz, F. Gama, and A. Ribeiro, "Graph neural networks: Architectures, stability and transferability," arXiv preprint arXiv:2008.01767, 2020, A fundamental study on Graph Neural Networks with analysis of their decentralized architectures, stability, and transferability that can act as theoretical bases for using GNNs to solve practical multi-robot coordination problems.

27. - E. Tolstaya, F. Gama, J. Paulos, G. Pappas, V. Kumar, and A. Ribeiro, "Learning decentralized controllers for robot swarms with graph neural networks," in Conference on Robot Learning, 2020, pp. 671-682, The first work that implements GNNs to learn decentralized controllers for multi-robot formation, e.g., flocking.

28. Q. Li, F. Gama, A. Ribeiro, and A. Prorok, "Graph neural networks for decentralized multi-robot path planning," arXiv preprint arXiv:1912.06095, 2019.

29. • Z. Wang and M. Gombolay, "Learning scheduling policies for multi-robot coordination with graph attention networks," IEEE Robotics and Automation Letters, vol. 5, no. 3, pp. 4509-4516, 2020, This study implements GNNs to learn real-time policies for multi-robot scheduling that is modeled as a combinatorial optimization problem.

30. J. Chen, A. Baskaran, Z. Zhang, and P. Tokekar, "Multi-agent reinforcement learning for persistent monitoring," arXiv preprint arXiv:2011.01129, 2020.
31. A. Prorok, "Graph neural networks for learning robot team coordination," arXiv preprint arXiv:1805.0373\%, 2018.

32. T. Zhang, W. Zhang, and M. M. Gupta, "Resilient robots: concept, review, and future directions," Robotics, vol. 6, no. 4, p. 22, 2017.

33. N. Bezzo, J. Weimer, M. Pajic, O. Sokolsky, G. J. Pappas, and I. Lee, "Attack resilient state estimation for autonomous robotic systems," in 2014 IEEE/RSJ International Conference on Intelligent Robots and Systems. IEEE, 2014, pp. 36923698.

34. N. Bezzo, J. Weimer, Y. Du, O. Sokolsky, S. H. Son, and I. Lee, "A stochastic approach for attack resilient uav motion planning," in 2016 American Control Conference (ACC). IEEE, 2016, pp. 1366-1372.

35. H. J. LeBlanc, H. Zhang, X. Koutsoukos, and S. Sundaram, "Resilient asymptotic consensus in robust networks," IEEE Journal on Selected Areas in Communications, vol. 31, no. 4, pp. 766-781, 2013.

36. V. Renganathan and T. Summers, "Spoof resilient coordination for distributed multi-robot systems," in 2017 International Symposium on Multi-Robot and Multi-Agent Systems (MRS). IEEE, 2017, pp. $135-141$.

37. D. Saldana, A. Prorok, M. F. Campos, and V. Kumar, "Triangular networks for resilient formations," in Distributed Autonomous Robotic Systems. Springer, 2018, pp. 147-159.

38. L. Guerrero-Bonilla, D. Saldana, and V. Kumar, "Design guarantees for resilient robot formations on lattices," IEEE Robotics and Automation Letters, vol. 4, no. 1, pp. 89-96, 2018.

39. D. Saldaña, L. Guerrero-Bonilla, and V. Kumar, "Resilient backbones in hexagonal robot formations," in Distributed Autonomous Robotic Systems. Springer, 2019, pp. 427-440.

40. L. Guerrero-Bonilla and V. Kumar, "Realization of $r$-robust formations in the plane using control barrier functions," IEEE Control Systems Letters, vol. 4, no. 2, pp. 343-348, 2019.

41. J. Usevitch and D. Panagou, "Resilient leaderfollower consensus to arbitrary reference values in time-varying graphs," IEEE Transactions on Automatic Control, vol. 65, no. 4, pp. 1755-1762, 2019.

42. —_ , "Resilient finite-time consensus: A discontinuous systems perspective," in 2020 American Control Conference (ACC). IEEE, 2020, pp. 3285-3290. 
43. D. Senejohnny, S. Sundaram, C. De Persis, and P. Tesi, "Resilience against misbehaving nodes in self-triggered coordination networks," in 2018 IEEE Conference on Decision and Control $(C D C)$. IEEE, 2018, pp. 2848-2853.

44. D. M. Senejohnny, S. Sundaram, C. De Persis, and P. Tesi, "Resilience against misbehaving nodes in asynchronous networks," Automatica, vol. 104, pp. 26-33, 2019.

45. X. Sun, R. Nambiar, M. Melhorn, Y. Shoukry, and P. Nuzzo, "Dos-resilient multi-robot temporal logic motion planning," in 2019 International Conference on Robotics and Automation (ICRA). IEEE, 2019, pp. 6051-6057.

46. A. Mitra and S. Sundaram, "Secure distributed observers for a class of linear time invariant systems in the presence of byzantine adversaries," in 2016 IEEE 55th Conference on Decision and Control $(C D C)$. IEEE, 2016, pp. 2709-2714.

47. A. Mitra, W. Abbas, and S. Sundaram, "On the impact of trusted nodes in resilient distributed state estimation of lti systems," in 2018 IEEE Conference on Decision and Control (CDC). IEEE, 2018, pp. 4547-4552.

48. A. Mitra and S. Sundaram, "Byzantine-resilient distributed observers for lti systems," Automatica, vol. 108, p. 108487, 2019.

49. L. Zhou and P. Tokekar, "An approximation algorithm for distributed resilient submodular maximization," in 2019 International Symposium on Multi-Robot and Multi-Agent Systems (MRS). IEEE, 2019, pp. 216-218.

50. L. Zhou, V. Tzoumas, G. J. Pappas, and P. Tokekar, "Distributed attack-robust submodular maximization for multi-robot planning," in 2020 IEEE International Conference on Robotics and Automation (ICRA). IEEE, 2020, to appear.

51. G. Shi, L. Zhou, and P. Tokekar, "Robust multiple-path orienteering problem: Securing against adversarial attacks," in 2020 Robotics: Science and Systems (RSS), 2020, to appear.

52. D. Shishika and V. Kumar, "Local-game decomposition for multiplayer perimeter-defense problem," in 2018 IEEE Conference on Decision and Control (CDC). IEEE, 2018, pp. 2093-2100.

53. D. Shishika, J. Paulos, M. R. Dorothy, M. A. Hsieh, and V. Kumar, "Team composition for perimeter defense with patrollers and defenders," in 2019 IEEE 58th Conference on Decision and Control (CDC). IEEE, 2019, pp. 7325-7332.

54. D. Shishika, J. Paulos, and V. Kumar, "Cooperative team strategies for multi-player perimeterdefense games," IEEE Robotics and Automation
Letters, vol. 5, no. 2, pp. 2738-2745, 2020.

55. R. K. Ramachandran, N. Fronda, and G. S. Sukhatme, "Resilience in multi-robot target tracking through reconfiguration," in 2019 IEEE/International Conference on Robotics and Automations (ICRA), Nov 2020, pp. 6518-6525.

56. R. K. Ramachandran, L. Zhou, J. A. Preiss, and G. S. Sukhatme, "Resilient coverage: Exploring the local-to-global trade-off," in 2020 IEEE/RSJ International Conference on Intelligent Robots and Systems (IROS), Oct 2020, to appear.

57. S. Mayya, D. Saldaña, and V. Kumar, "Resilient task allocation in heterogeneous multi-robot systems," arXiv preprint arXiv:2009.04593, 2020.

58. R. K. Ramachandran, P. Pierpaoli, M. Egerstedt, and G. S. Sukhatme, "Resilient monitoring in heterogeneous multi-robot systems through network reconfiguration," arXiv preprint arXiv:2008.01321, 2020.

59. K.-K. Oh, M.-C. Park, and H.-S. Ahn, "A survey of multi-agent formation control," Automatica, vol. 53, pp. 424-440, 2015.

60. P. Tokekar, V. Isler, and A. Franchi, "Multitarget visual tracking with aerial robots," in 2014 IEEE/RSJ International Conference on Intelligent Robots and Systems (IROS). IEEE, 2014, pp. 3067-3072.

61. N. Atanasov, J. Le Ny, K. Daniilidis, and G. J. Pappas, "Information acquisition with sensing robots," in 2014 IEEE International Conference on Robotics and Automation (ICRA), 2014, pp. 6447-6454.

62. L. Zhou and P. Tokekar, "Active target tracking with self-triggered communications," in 2017 IEEE International Conference on Robotics and Automation (ICRA). IEEE, 2017, pp. 2117-2123.

63. — , "Active target tracking with self-triggered communications in multi-robot teams," IEEE Transactions on Automation Science and Engineering, vol. 16, no. 3, pp. 1085-1096, 2018.

64. — - "Sensor assignment algorithms to improve observability while tracking targets," IEEE Transactions on Robotics, vol. 35, no. 5, pp. 1206-1219, 2019.

65. M. Michini, M. A. Hsieh, E. Forgoston, and I. B. Schwartz, "Robotic tracking of coherent structures in flows," IEEE Transactions on Robotics, vol. 30, no. 3, pp. 593-603, 2014.

66. V. Kumar and N. Michael, "Opportunities and challenges with autonomous micro aerial vehicles," The International Journal of Robotics Research, vol. 31, no. 11, pp. 1279-1291, 2012. 
67. G. L. Nemhauser, L. A. Wolsey, and M. L. Fisher, "An analysis of approximations for maximizing submodular set functions-i," Mathematical Programming, vol. 14, no. 1, pp. 265-294, 1978.

68. M. L. Fisher, G. L. Nemhauser, and L. A. Wolsey, "An analysis of approximations for maximizing submodular set functions-ii," in Polyhedral Combinatorics. Springer, 1978, pp. 73-87.

69. - - V. Tzoumas, K. Gatsis, A. Jadbabaie, and G. J. Pappas, "Resilient monotone submodular function maximization," in 2017 IEEE 56th Annual Conference on Decision and Control (CDC). IEEE, 2017, pp. 1362-1367, A fundamental study that formulates a resilient submodular maximization problem and presents a polynomial-time and provably close-tooptimal algorithm for solving it. The findings from this paper are generic and can be applied to many multi-robot applications where a team of robots aims to optimize a submodular objective in adversarial environments.

70. D. Song, C.-Y. Kim, and J. Yi, "Simultaneous localization of multiple unknown and transient radio sources using a mobile robot," IEEE Transactions on Robotics, vol. 28, no. 3, pp. 668-680, 2012.

71. O. Peltzer, K. Brown, M. Schwager, M. J. Kochenderfer, and M. Sehr, "Stt-cbs: A conflictbased search algorithm for multi-agent path finding with stochastic travel times," arXiv preprint arXiv:2004.08025, 2020.

72. E. Yel, T. X. Lin, and N. Bezzo, "Self-triggered adaptive planning and scheduling of uav operations," in 2018 IEEE International Conference on Robotics and Automation (ICRA). IEEE, 2018, pp. $7518-7524$.

73. M. Toubeh and P. Tokekar, "Risk-aware planning by confidence estimation using deep learning-based perception," arXiv preprint arXiv:1910.00101, 2019.

74. • Y. Chow, A. Tamar, S. Mannor, and M. Pavone, "Risk-sensitive and robust decision-making: a cvar optimization approach," in Advances in Neural Information Processing Systems, 2015, pp. 15221530, This study presents the first approximate value-iteration algorithm with error guarantees for solving CVaR MDPs.

75. Y. Chow, M. Ghavamzadeh, L. Janson, and M. Pavone, "Risk-constrained reinforcement learning with percentile risk criteria," The Journal of Machine Learning Research, vol. 18, no. 1, pp. 6070-6120, 2017.
76. • A. Majumdar and M. Pavone, "How should a robot assess risk? towards an axiomatic theory of risk in robotics," in Robotics Research. Springer, 2020, pp. 75-84, This study analyzes and discusses how should a robot quantifies risk and what constitutes a "good" risk measure toward ensuring safety for robots performing under uncertainty.

77. D. Fridovich-Keil, A. Bajcsy, J. F. Fisac, S. L. Herbert, S. Wang, A. D. Dragan, and C. J. Tomlin, "Confidence-aware motion prediction for real-time collision avoidance," The International Journal of Robotics Research, vol. 39, no. 2-3, pp. 250-265, 2020.

78. S. Singh, Y. Chow, A. Majumdar, and M. Pavone, "A framework for time-consistent, risk-sensitive model predictive control: Theory and algorithms," IEEE Transactions on Automatic Control, vol. 64, no. 7, pp. 2905-2912, 2018.

79. F. Yang and N. Chakraborty, "Algorithm for optimal chance constrained knapsack problem with applications to multi-robot teaming," in 2018 IEEE International Conference on Robotics and Automation (ICRA). IEEE, 2018, pp. 1043-1049.

80. — - "Chance constrained simultaneous path planning and task assignment for multiple robots with stochastic path costs," in 2020 IEEE International Conference on Robotics and Automation (ICRA). IEEE, 2020, pp. 6661-6667.

81. S. Jorgensen, R. H. Chen, M. B. Milam, and M. Pavone, "The team surviving orienteers problem: routing teams of robots in uncertain environments with survival constraints," Autonomous Robots, vol. 42, no. 4, pp. 927-952, 2018.

82. J. Lacotte, M. Ghavamzadeh, Y. Chow, and M. Pavone, "Risk-sensitive generative adversarial imitation learning," in The 22nd International Conference on Artificial Intelligence and Statistics. PMLR, 2019, pp. 2154-2163.

83. C. Nam and D. A. Shell, "Analyzing the sensitivity of the optimal assignment in probabilistic multi-robot task allocation," IEEE Robotics and Automation Letters, vol. 2, no. 1, pp. 193-200, 2016.

84. H. Zhu and J. Alonso-Mora, "B-uavc: Buffered uncertainty-aware voronoi cells for probabilistic multi-robot collision avoidance," in 2019 International Symposium on Multi-Robot and MultiAgent Systems (MRS). IEEE, 2019, pp. 162-168.

85. M. da Silva Arantes, C. F. M. Toledo, B. C. Williams, and M. Ono, "Collision-free encoding for chance-constrained nonconvex path planning," IEEE Transactions on Robotics, vol. 35, no. 2, pp. 
433-448, 2019.

86. A. Wang, A. Jasour, and B. C. Williams, "Nongaussian chance-constrained trajectory planning for autonomous vehicles under agent uncertainty," IEEE Robotics and Automation Letters, vol. 5, no. 4, pp. 6041-6048, 2020.

87. V. Indelman, "Cooperative multi-robot belief space planning for autonomous navigation in unknown environments," Autonomous Robots, vol. 42, no. 2, pp. 353-373, 2018.

88. M. J. Kochenderfer, Decision making under uncertainty: theory and application. MIT press, 2015.

89. - C. Amato, G. Konidaris, A. Anders, G. Cruz, J. P. How, and L. P. Kaelbling, "Policy search for multi-robot coordination under uncertainty," The International Journal of Robotics Research, vol. 35, no. 14, pp. 1760-1778, 2016, The study presents a new MacDec-POMDP planning algorithm that utilizes macro-actions to solve significantly larger problems than existing Dec-POMDP planners.

90. S. Omidshafiei, A.-A. Agha-Mohammadi, C. Amato, S.-Y. Liu, J. P. How, and J. Vian, "Decentralized control of multi-robot partially observable markov decision processes using belief space macro-actions," The International Journal of Robotics Research, vol. 36, no. 2, pp. 231-258, 2017.

91. C. Amato, G. Konidaris, L. P. Kaelbling, and J. P. How, "Modeling and planning with macro-actions in decentralized pomdps," Journal of Artificial Intelligence Research, vol. 64, pp. 817-859, 2019.

92. S. Omidshafiei, J. Pazis, C. Amato, J. P. How, and J. Vian, "Deep decentralized multi-task multiagent reinforcement learning under partial observability," arXiv preprint arXiv:1703.06182, 2017.

93. M. Toubeh, L. Zhou, and P. Tokekar, "Risk-aware path planning and assignment with uncertainty extraction from deep learning," in 2019 Northeast Robotics Colloquium (NERC), accepted as poster presentation.

94. V. D. Sharma, M. Toubeh, L. Zhou, and P. Tokekar, "Risk-aware planning and assignment for ground vehicles using uncertain perception from aerial vehicles," in 2020 IEEE/RSJ International Conference on Intelligent Robots and Systems (IROS). IEEE, 2020, to appear.

95. R. T. Rockafellar and S. Uryasev, "Optimization of conditional value-at-risk," Journal of Risk, vol. 2, pp. 21-42, 2000.

96. T. Maehara, "Risk averse submodular utility maximization," Operations Research Letters, vol. 43, no. 5, pp. 526-529, 2015.
97. N. Ohsaka and Y. Yoshida, "Portfolio optimization for influence spread," in Proceedings of the 26th International Conference on World Wide Web. International World Wide Web Conferences Steering Committee, 2017, pp. 977-985.

98. B. Wilder, "Risk-sensitive submodular optimization," in Proceedings of the 32nd AAAI Conference on Artificial Intelligence, 2018.

99. L. Zhou and P. Tokekar, "Risk-aware submodular optimization for multi-robot coordination," IEEE Transactions on Robotics, submitted.

100. R. Balasubramanian, L. Zhou, P. Tokekar, and P. Sujit, "Risk-aware submodular optimization for multi-objective travelling salesperson problem," arXiv preprint arXiv:2011.01095, 2020.

101. S. M. LaValle, Planning algorithms. Cambridge university press, 2006.

102. P. E. Hart, N. J. Nilsson, and B. Raphael, "A formal basis for the heuristic determination of minimum cost paths," IEEE transactions on Systems Science and Cybernetics, vol. 4, no. 2, pp. 100-107, 1968.

103. S. Karaman and E. Frazzoli, "Sampling-based algorithms for optimal motion planning," The international journal of robotics research, vol. 30 , no. 7 , pp. 846-894, 2011.

104. G. A. Hollinger, A. A. Pereira, J. Binney, T. Somers, and G. S. Sukhatme, "Learning uncertainty in ocean current predictions for safe and reliable navigation of underwater vehicles," Journal of Field Robotics, vol. 33, no. 1, pp. 47-66, 2016.

105. G. E. Monahan, "State of the art-a survey of partially observable markov decision processes: theory, models, and algorithms," Management science, vol. 28, no. 1, pp. 1-16, 1982.

106. M. Liu, C. Amato, E. P. Anesta, J. D. Griffith, and J. P. How, "Learning for decentralized control of multiagent systems in large, partiallyobservable stochastic environments." in $A A A I$, 2016, pp. 2523-2529.

107. S. Choudhury, J. K. Gupta, M. J. Kochenderfer, D. Sadigh, and J. Bohg, "Dynamic multi-robot task allocation under uncertainty and temporal constraints," arXiv preprint arXiv:2005.13109, 2020.

108. F. Gama, E. Tolstaya, and A. Ribeiro, "Graph neural networks for decentralized controllers," arXiv preprint arXiv:2003.10280, 2020.

109. Y.-C. Liu, J. Tian, N. Glaser, and Z. Kira, "When2com: Multi-agent perception via communication graph grouping," in Proceedings of the IEEE/CVF Conference on Computer Vision and 
Pattern Recognition, 2020, pp. 4106-4115.

110. Y.-C. Liu, J. Tian, C.-Y. Ma, N. Glaser, C.-W. Kuo, and Z. Kira, "Who2com: Collaborative perception via learnable handshake communication," arXiv preprint arXiv:2003.09575, 2020.

111. F. Gama, J. Bruna, and A. Ribeiro, "Stability properties of graph neural networks," arXiv preprint arXiv:1905.04497, 2019.

112. A. Khan, E. Tolstaya, A. Ribeiro, and V. Kumar, "Graph policy gradients for large scale robot control," in Conference on Robot Learning, 2020, pp. 823-834.

113. A. Khan, V. Kumar, and A. Ribeiro, "Graph policy gradients for large scale unlabeled motion planning with constraints," arXiv preprint arXiv:1909.10704, 2019.

114. R. S. Sutton and A. G. Barto, Reinforcement learning: An introduction. MIT press, 2018.

115. S. Liu, G. Lever, J. Merel, S. Tunyasuvunakool, N. Heess, and T. Graepel, "Emergent coordination through competition," arXiv preprint arXiv:1902.07151, 2019.

116. J. Blumenkamp and A. Prorok, "The emergence of adversarial communication in multiagent reinforcement learning," arXiv preprint arXiv:2008.02616, 2020.

117. A. Kurakin, I. Goodfellow, and S. Bengio, "Adversarial examples in the physical world," in 2017 International Conference on Learning Representation (ICLR) (Workshop), 2017.

118. K. Eykholt, I. Evtimov, E. Fernandes, B. Li, A. Rahmati, C. Xiao, A. Prakash, T. Kohno, and D. Song, "Robust physical-world attacks on deep learning visual classification," in Proceedings of the IEEE Conference on Computer Vision and Pattern Recognition, 2018, pp. 1625-1634.

119. A. Madry, A. Makelov, L. Schmidt, D. Tsipras, and A. Vladu, "Towards deep learning models resistant to adversarial attacks," arXiv preprint arXiv:1706.06083, 2017.

120. A. Athalye, N. Carlini, and D. Wagner, "Obfuscated gradients give a false sense of security: Circumventing defenses to adversarial examples," arXiv preprint arXiv:1802.00420, 2018.

121. F. Tramèr, A. Kurakin, N. Papernot, I. Goodfellow, D. Boneh, and P. McDaniel, "Ensemble adversarial training: Attacks and defenses," arXiv preprint arXiv:1705.07204, 2017. 